\title{
Single-cell RNA sequencing reveals heterogeneous tumor and immune cell populations in early-stage lung adenocarcinomas harboring EGFR mutations
}

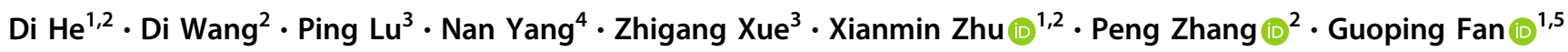

Received: 14 February 2020 / Revised: 3 October 2020 / Accepted: 15 October 2020 / Published online: 3 November 2020

(c) The Author(s) 2020. This article is published with open access

\begin{abstract}
Lung adenocarcinoma (LUAD) harboring EGFR mutations prevails in Asian population. However, the inter-patient and intra-tumor heterogeneity has not been addressed at single-cell resolution. Here we performed single-cell RNA sequencing (scRNA-seq) of total 125,674 cells from seven stage-I/II LUAD samples harboring EGFR mutations and five tumor-adjacent lung tissues. We identified diverse cell types within the tumor microenvironment (TME) in which myeloid cells and T cells were the most abundant stromal cell types in tumors and adjacent lung tissues. Within tumors, accompanied by an increase in $\mathrm{CD}_{1 \mathrm{C}^{+}}$dendritic cells, the tumor-associated macrophages (TAMs) showed pro-tumoral functions without signature gene expression of defined M1 or M2 polarization. Tumor-infiltrating T cells mainly displayed exhausted and regulatory T-cell features. The adenocarcinoma cells can be categorized into different subtypes based on their gene expression signatures in distinct pathways such as hypoxia, glycolysis, cell metabolism, translation initiation, cell cycle, and antigen presentation. By performing pseudotime trajectory, we found that $E L F 3$ was among the most upregulated genes in more advanced tumor cells. In response to secretion of inflammatory cytokines (e.g., IL1B) from immune infiltrates, ELF3 in tumor cells was upregulated to trigger the activation of PI3K/Akt/NF- $\mathrm{kB}$ pathway and elevated expression of proliferation and anti-apoptosis genes such as $B C L 2 L 1$ and $C C N D 1$. Taken together, our study revealed substantial heterogeneity within early-stage LUAD harboring EGFR mutations, implicating complex interactions among tumor cells, stromal cells and immune infiltrates in the TME.
\end{abstract}

These authors contributed equally: Xianmin Zhu, Peng Zhang, Guoping Fan

Supplementary information The online version of this article (https:// doi.org/10.1038/s41388-020-01528-0) contains supplementary material, which is available to authorized users.

$\triangle$ Xianmin Zhu

xianminzhu@hotmail.com

$\triangle$ Peng Zhang

zhangpeng1121@aliyun.com

$\triangle$ Guoping Fan

gfan@mednet.ucla.edu

1 Shanghai Institute for Advanced Immunochemical Studies, ShanghaiTech University, Shanghai 201210, China

2 Shanghai Pulmonary Hospital, Department of Thoracic Surgery, School of Life Sciences and Technology, Tongji University,

\section{Introduction}

Lung cancer is the leading cause of cancer-related deaths worldwide. Non-small cell lung cancer (NSCLC) accounts for $\sim 85 \%$ of new lung cancer cases and has a poor 5-year survival rate below $16 \%$ [1]. In fact, lung adenocarcinoma (LUAD) becomes the most common pathological subtype of NSCLC, with increased frequency in young women and

\section{Shanghai 200433, China}

3 Translational Center for Stem Cell Research, Tongji Hospital, Department of Regenerative Medicine, Tongji University School of Medicine, Shanghai 200065, China

4 PharmaLegacy Laboratories (Shanghai) Co, Zhangjiang HighTech Park Ltd, Building 7, 388 Jialilue Road, Shanghai 201203, China

5 Department of Human Genetics, David Geffen School of Medicine, University of California Los Angeles, Los Angeles, CA 90095, USA 
never smokers. Of note, EGFR mutations in LUAD are generally high in Asian women [2]. The most common EGFR mutations include deletions in exon 19 and L858R point mutation in exon 21. LUAD harboring these EGFR mutations is sensitive to tyrosine kinase inhibitor (TKI) treatment, but eventually develops acquired resistance after a year or so of progression-free period $[3,4]$.

Besides diverse pathological characteristics, NSCLC exhibits inter-patient and intra-tumoral heterogeneity in both tumor cells and microenvironments [5]. The tumor microenvironment (TME) consists of many cell types including immune infiltrates (e.g., mononuclear phagocytes, $\mathrm{T}$ cells, dendritic cells, B cells, and mast cells), cancerassociated fibroblasts (CAFs), and vascular endothelial cells. The TME components vary markedly among different tumors and play crucial roles in tumor initiation, progression, and metastasis [6, 7]. By modulating tumor-infiltrating immune cells, novel immunotherapies have already achieved great success in clinic. For instance, blocking the immune checkpoint molecules such as CTLA-4 and PD-1 can activate anti-tumoral cytotoxicity of $\mathrm{T}$ cells $[8,9]$. Adoptive $\mathrm{T}$-cell therapies using engineered $\mathrm{T}$ cells with chimeric antigen receptors (CARs) also hold great potential in clinical application [10]. However the efficacy of the immunotherapies is inconsistent among different patients, which may be resulted from heterogeneity of tumor cells and their microenvironments.

Recently, single-cell RNA-sequencing (scRNA-seq) technology has been used to study heterogeneous gene expression of different tissue samples. For example, droplet-based scRNA-seq methods with high throughput [11-15] have fueled the investigations of tumor microenvironment of many cancer types such as acute myeloid leukemia (AML) [12], breast cancer [16], pancreatic ductal adenocarcinoma [17], NSCLC [18]. In this study, we performed scRNAseq analysis of early-stage (stage I/II) LUAD from seven patients carrying EGFR mutations (Table 1). By comparing cellular heterogeneity of tumor cells with adjacent control lung tissues, we investigated the complex interactions among different cell types including tumor cells and other major cell components of the TME.

\section{Results}

\section{scRNA-seq of seven LUAD samples harboring EGFR mutations}

We collected the resected tumor tissues from 7 untreated early-stage (stage I/II) LUAD patients (LUAD1 to 7) who all survived lobectomy surgery up to two years. All of them carried the most common EGFR active mutations, i.e., L858R point mutation and deletions in exon 19 (Table 1).
For comparison, we also profiled cells from 5 tumoradjacent normal lung tissues matching LUAD1-LUAD5, respectively. Immediately after resection, tumors and normal lung tissues were collected with enough aliquots for scRNA-seq and immunohistological evaluation. We then prepared single-cell suspensions from the tissues and constructed scRNA-seq libraries following 10X Genomics single-cell $3^{\prime}$ RNA library construction and sequencing pipeline (Fig. 1a). We obtained about 4.3 billion unique transcripts from 158,306 cells in which 1147 genes per cell were detected. After data processing and normalization, we obtained 125,674 cells for subsequent analysis (Supplementary Table S3) by removing potential doublets and the cells with poor quality (too few transcripts or too much mitochondria-derived RNA). We performed unsupervised clustering of the single cells from all seven tumor samples and five matched lung tissues and retrieved 32 distinct clusters (Fig. 1b) which were visualized by Uniform Manifold Approximation and Projection for Dimension Reduction (UMAP). To identify different cell types, we analyzed the expression of the canonical markers in each cluster (Supplementary Fig. S1, Supplementary Table S4) as well as the enrichment of differentially expressed genes (DEGs) (Fig. 1c), allowing us to categorize these clusters into tumor cells, bronchial/alveolar epithelial cells, myeloid cells, T lymphocytes, B lymphocytes, cancer-associated fibroblasts, endothelial cells and mast cells (Fig. 1b, c). The scRNA-seq data showed that myeloid cells and T lymphocytes accounted for the majority of the stromal cells, although the fractions of each cell type varied in different samples (Supplementary Fig. S2a). Consistently, immunohistochemistry also showed presence of many macrophages and $\mathrm{T}$ lymphocytes in tumor samples (Supplementary Fig. S2b).

\section{Tumor associate macrophages (TAMs) are in intermediate polarization states with pro-tumoral functions}

Since the immune components could exert crucial influence on tumorigenesis and progression [19, 20], we first sought to investigate the features of the immune infiltrates in earlystage LUAD samples. As previously stated, myeloid cells account for the most abundant immune infiltrates in both tumors ( 24\%-70\%) and normal lungs ( $32 \%-64 \%)$ in our experiment (Supplementary Fig S2). We grouped all the myeloid cells into 14 heterogeneous subclusters and found that the majority $(\sim 90 \%)$ of the myeloid cells were CD68expressing macrophages (Fig. 2a, b, Supplementary Fig. S3a). CD11B (ITGAM) was not highly expressed in the myeloid cells (data not shown). Increased dendritic cells and decreased granulocytes were found in the TME compared to the tumor-adjacent normal tissues (Fig. 2a, 
Table 1 Information of the patients for scRNA-seq.

\begin{tabular}{|c|c|c|c|c|c|c|c|c|c|}
\hline Sample & Patient & Sex & Age & TNM stage & Pathological stage & Driver mutation & Smoking status & COPD & Metastases \\
\hline T0418 & LUAD1 & $\mathrm{F}$ & 48 & T2aNOM0 & IB & EGFR 19del & Never & No & No \\
\hline T0422 & LUAD2 & M & 61 & T1bN1M0 & IIA & EGFR L858R & Active & No & No \\
\hline T0423 & LUAD3 & $\mathrm{F}$ & 58 & T1bN0M0 & IA & EGFR 19del & Active & Yes & No \\
\hline T0424 & LUAD4 & M & 75 & T1bN0M0 & IA & EGFR 19del & Never & No & a \\
\hline T0425 & LUAD5 & $\mathrm{F}$ & 56 & T2aN1M0 & IIA & EGFR 19del & Never & No & No \\
\hline $\mathrm{T} 102$ & LUAD6 & $\mathrm{F}$ & 53 & T1cN0M0 & IA 3 & EGFR L858R & Never & No & No \\
\hline S1028531 & LUAD7 & $\mathrm{F}$ & 57 & T2aN0M0 & IB & EGFR 19del & Never & No & No \\
\hline
\end{tabular}

${ }^{a}$ Mediastinal and lymph nodes are swelling, and metastases are confirmed in clinical follow-up.

a
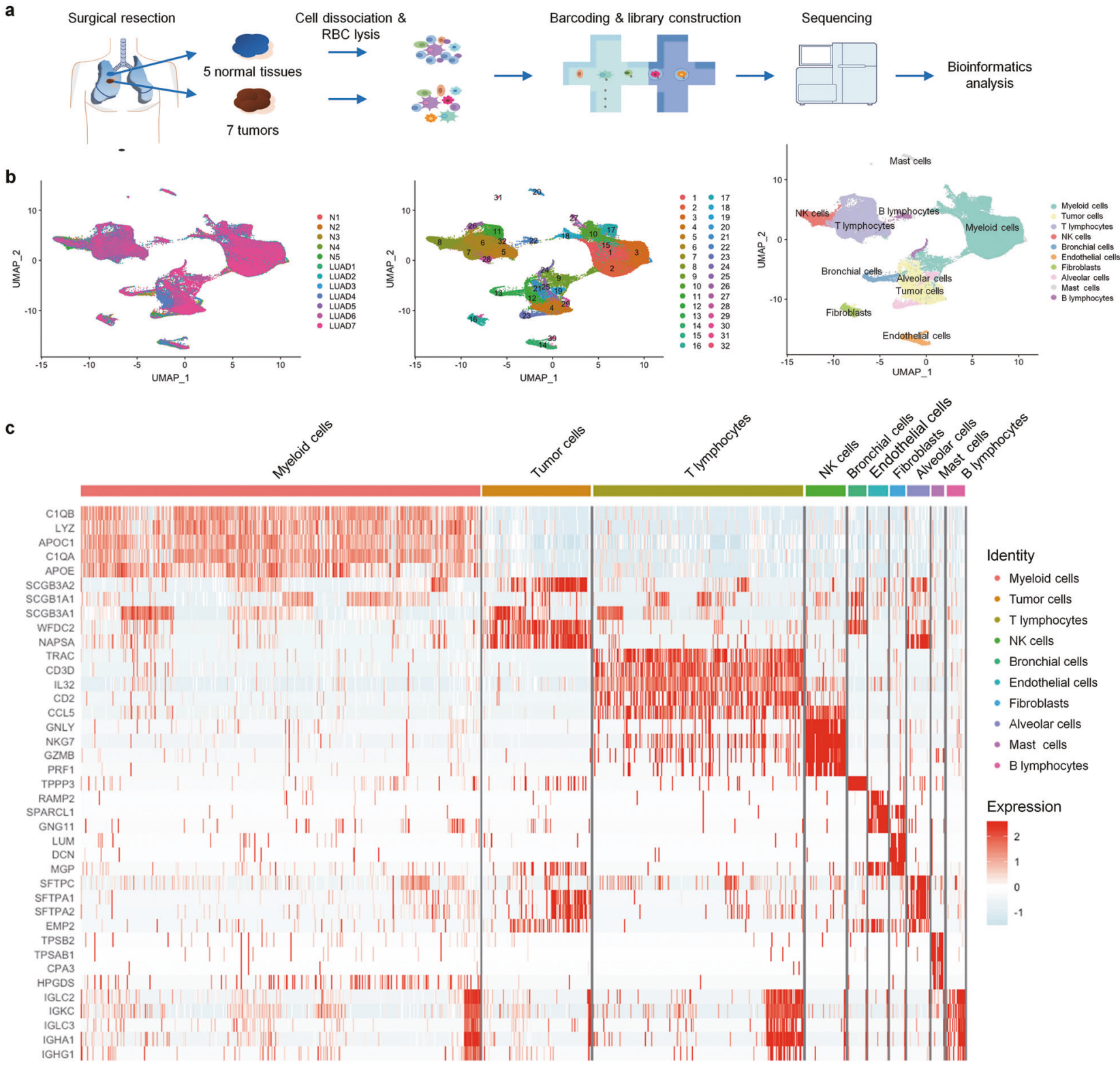

Identity

- Myeloid cells

- Tumor cells

- Tlymphocytes

- NK cells

- Bronchial cells

- Endothelial cells

- Fibroblasts

- Alveolar cells

- Mast cells

- B lymphocytes

Expression

Fig. 1 scRNA-seq of control tissues and LUAD samples harboring EGFR mutations. a Overview of the workflow. b UMAP plot of 125,674 profiled cells from all the samples (both LUAD tumor and tumor-adjacent normal tissues). Left: different color labeled for different samples. Middle: different color labeled for 32 clusters, respectively. Right: different color labeled for different cell types. c Heatmap of the top 5 DEGs $(p<=0.05$, fold change $>=1.5$ ) in each cell type. 

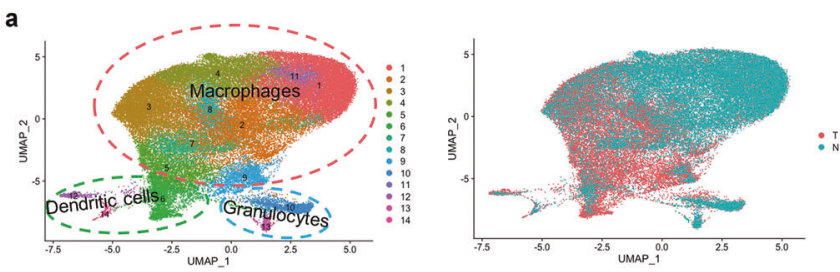

b
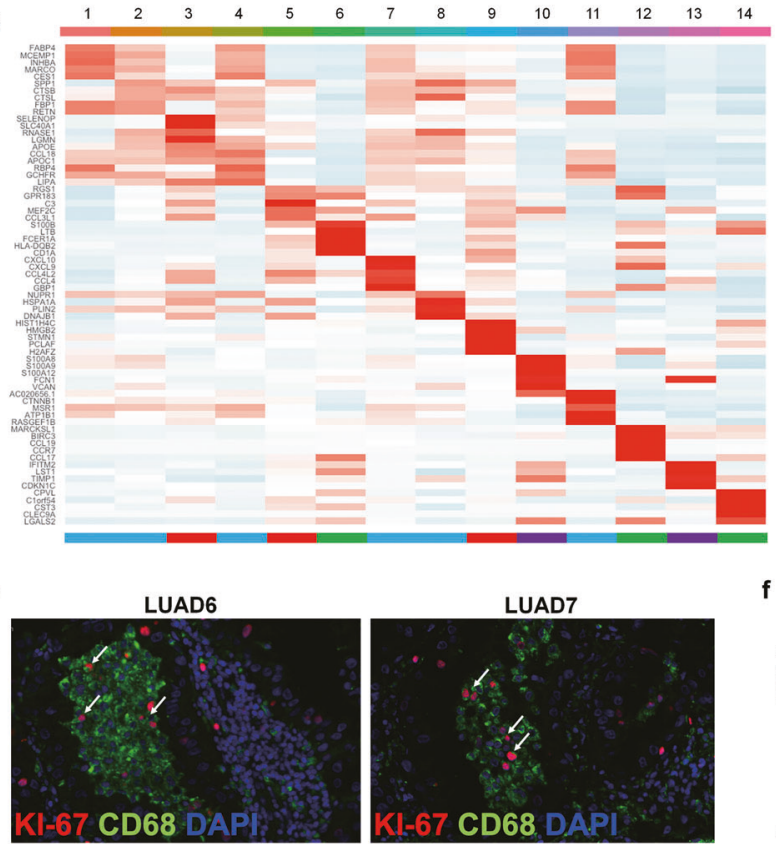

g
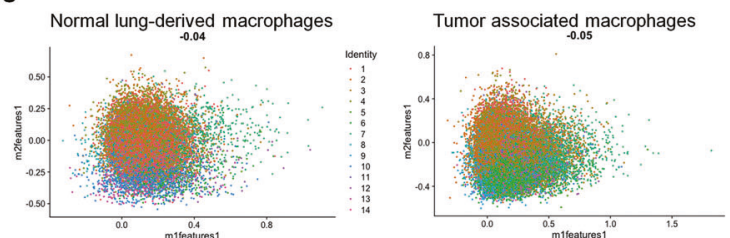

Fig. 2 Tumor-associated macrophages (TAMs) exhibit protumoral functions and are at intermediate polarization states. a UMAP plots with cells color-coded for 14 myeloid cell subclusters (left) and different tissue origins (right). b Heatmap showing the average expression of top 5 DEGs in each macrophage sub-cluster. c Scatter plot showing the top differentially expressed genes between tumor-associated macrophages and normal lung-derived macrophages. Red circle highlighted the two most highly expressed genes, SPP1 and $A P O E$, in tumor-associated macrophages. $\mathbf{d}$ Bar plot of representative enriched GO BP terms of DEGs in TAMs. e Immunostaining with anti- CD68 and KI67 antibodies identified the existence of

Supplementary Fig. S3). Compared to macrophages in the normal tissues, the TAMs have high expression of $A P O E$ (Fold change $=23.65909118, \quad$ adjusted $\quad p$ value $=$ $2.225074 \mathrm{e}-308$ ) and SPP1 (Fold change $=17.40700342$, adjusted $p$ value $=2.225074 \mathrm{e}-308$ ) (Fig. 2c), which were reported to promote tumor cell growth and invasiveness $[21,22]$. The DEGs enriched in TAMs were related to pathways such as extracellular matrix disassembly, response to hypoxia, positive regulation of cholesterol efflux and

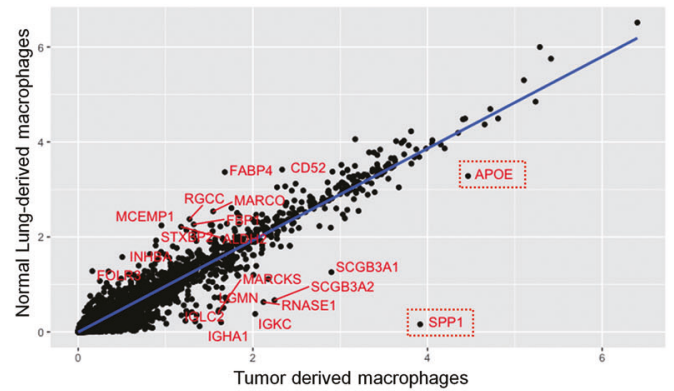

d

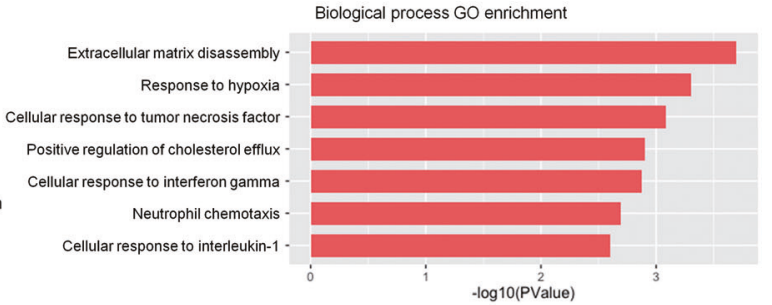

Macrophages

TAMs

Granulocytes
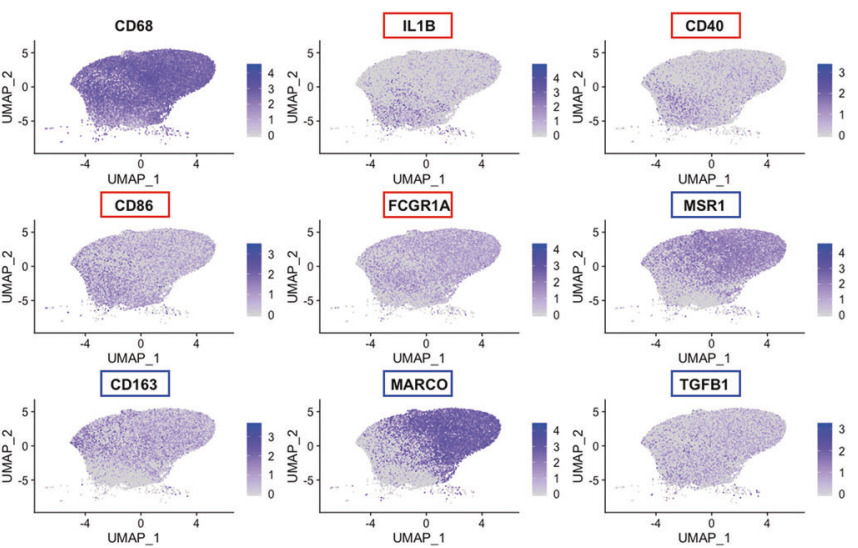

proliferating macrophages in the LUAD tumor tissues. f UMAP showing the expression of canonical M1 (red) and M2 (blue) feature genes in all the macrophage (CD68-expressing) cells. $\mathbf{g}$ Scatterplots of normalized weighted mean expression of M1 and M2 signature genes (Supplementary Table S4) in each cell (dot) from all the macrophages derived from tumor samples (top) and normal lung tissues (bottom). The plots showing that TAMs and normal lung-derived macrophages express both M1 and M2 signature genes (Pearson correlation coefficient $=0.16$ ), indicating they possess both polarized features and in various intermediate polarized states between M1 and M2 states.

response to TNF and IL1B (Fig. 2d), implying that they may promote cell migration, angiogenesis [23], tumor progression [24] and inflammation. Interestingly, we identified a population of TAMs (Cluster 8 in Fig. 2b) which had high expression of cell-cycle related genes such as NUPR1 $($ LogFoldChange $=1.102$, adjusted $p$ value $=$ 2.225074e-308), STMN1 (LogFoldChange $=1.69$, adjusted $p$ value $=2.225074 \mathrm{e}-308)$ and MKI67 (LogFoldChange $=1.075131675$, adjusted $p$ value $=2.225074 \mathrm{e}-308$ ) 
The existence of these proliferative macrophages was then verified by IHC using anti-CD68 and -KI67 antibodies (Fig. 2e).

Previous studies suggested that TAMs in tumors were phenotypically similar to anti-inflammatory M2-polarized macrophages [25]. To understand the polarization of TAMs in early-stage LUAD, we examined the expression of the conventional classic activated macrophage (M1) and alternative activated macrophage (M2) signature genes in these clusters (Fig. 2f, Supplementary Table S1). Interestingly, the macrophages in both tumors and normal lung tissues did not show exclusive M1 or M2 signature. Instead, they had similar expression level of both M1 and M2 signature genes (Fig. 2g). Taken together, while the TAMs in early-stage LUAD appear to express genes promoting tumorigenesis, they do not have distinct M1 and M2 polarization yet.

\section{Early-stage LUAD has immunosuppressive T lymphocytes and dendritic cell populations}

$\mathrm{T}$ cells account for $\sim 20-60 \%$ of all the non-malignant cell populations (Supplementary Fig. S2). Non-biased clustering of all the $\mathrm{T}$ cells did not show any different clusters of $\mathrm{T}$ cells between the tumor samples and normal tissues (Fig. 3a). So we decided to check the expression of the T-cell subtype markers in each cluster. We found that the tumorinfiltrating $\mathrm{T}$ cells highly expressed regulatory and exhausted markers, such as TIGIT (LogFoldChange $=$ 0.258224344 , adjusted $p$ value $=2.75 \mathrm{e}-143), \quad L A Y N$ (LogFoldChange $=0.071034001$, adjusted $p$ value $=1.90 \mathrm{e}$ -12), FOXP3 (LogFoldChange $=0.082017301$, adjusted $p$ value $=7.92 \mathrm{e}-13)$ and CTLA4 (LogFoldChange $=$ 0.101004531 , adjusted $p$ value $=4.63 \mathrm{e}-13$ ), while the $\mathrm{T}$ cells in normal tissues highly expressed effector and naïve T-cell markers (Fig. 3b, c, Supplementary Fig. S4). We also identified a subtype of proliferative T cells (Cluster 7 with high KI67 expression) which displayed both effector T-cell features (e.g., GZMA) and dysfunctional markers such as LAG3, TIGIT, and PD-1, indicating compromised tumor cytotoxic activity (Fig. 3d).

The abundance of subtypes of $\mathrm{T}$ cells in the tumor microenvironment is strongly associated with prognosis in response to checkpoint blockade therapies [26]. T-cell infiltration and differentiation can be influenced by many intrinsic properties of the tumors such as local environment of cytokines, chemokines, and the presence of other immune cells including dendritic cells (DCs) in the TME [27]. There is a subset of tumor-derived macrophages highly expressing $\mathrm{T}$ lymphocyte chemotactic genes CXCL9, CXCL10, and CXCL11 (LogFoldChange = $0.4858127,0.7869661$, and 0.3042152 respectively; all adjusted $p$ value $=2.225074 \mathrm{e}-308$ vs. normal lung-derived myeloid cells; Supplementary Fig. S5). As known, DCs play a vital role in mediating T-cell chemotaxis, differentiation, and activation. In our dataset, we found that the DCs in LUAD were mainly $\mathrm{CD}^{+} \mathrm{C}^{+}$, implying their function in inhibition of effector $\mathrm{T}$ cells and promotion of regulatory $\mathrm{T}$ cells (Fig. 3d, e). All together, our results suggested that the early stage LUAD has an immunosuppressive TME, in which $\mathrm{T}$ cells differentiate towards the regulatory and exhausted subtypes accompanied by increased presence of $\mathrm{CD}_{1 C^{+}}$DCs.

\section{Tumor cells within early-stage LUAD have distinct expression signatures}

To further verify our annotation of malignant and nonmalignant cells in the tumor tissues, we inferred large-scale copy number variations (CNVs) from expression intensity of 15,414 genes (Supplementary Table S5) across each chromosome of potential malignant cells using the annotated lung epithelial cells in tumor-adjacent tissues as background control. The cells with aberrant CNVs were identified as malignant cells (Fig. 4a, Supplementary Fig. S6), which were further grouped into 8 clusters based on the functional enrichment analysis of their DEGs (Fig. 4b). These clusters were enriched in the pathways such as hypoxia, glycolysis, oxidative phosphorylation, translation initiation, cell cycle, and antigen presentation, respectively (Fig. 4c, Supplementary Fig. S7). In K-Ras-induced mouse LUAD, tumor cells could arise from AT2 cells in alveolar and Clara cells in the bronchioles [28, 29]. However, we did not see high expression of the classic AT2 cell marker SFTPC or Clara cell marker SCGB1Al in the tumor cell, except in a population of cluster 3 (Supplementary Fig. S8). We then examined the expression of conanical markers in tumor cells compared to the normal lung epithelial cells. We found that the Clara cell progenitor marker SCGB3A2 $[30,31]($ LogFoldChange $=16.21089$, adjusted $p$ value $=$ 9.088149e-93) and alveolar epithelial progenitor (AEP) marker TM4SF1 [32] (LogFoldChange $=0.9938625$, adjusted $p$ value $=1.553989 \mathrm{e}-104)$ were upregulated in tumor cells (Fig. 4d, e, Supplmentary Fig. S9). As they highly expressed both proximal and distal epithelial progenitor markers, the tumor cells were composed of heterogeneous populations with expression profiles of diverse cell lineages.

\section{Pseudotime trajectory analysis shows that ELF3 is upregulated in more advanced tumor cells}

As described above, our results revealed different tumor cell clusters with DEGs enriched in distinct pathways, implying progressional heterogeneity among them. We then asked if the single-cell transcriptome could gain temporal information of differentiation states during LUAD progression. 

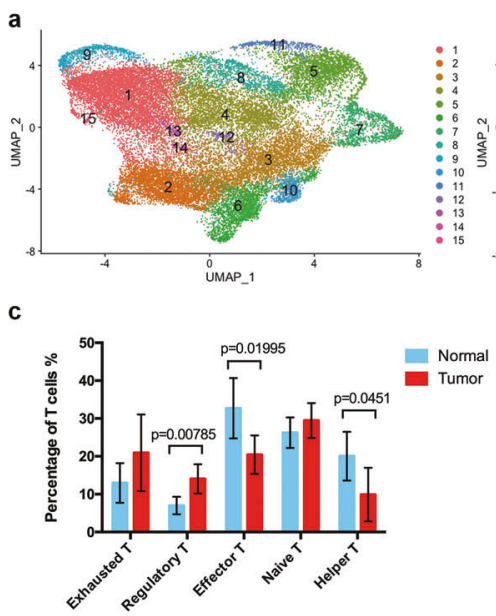

e
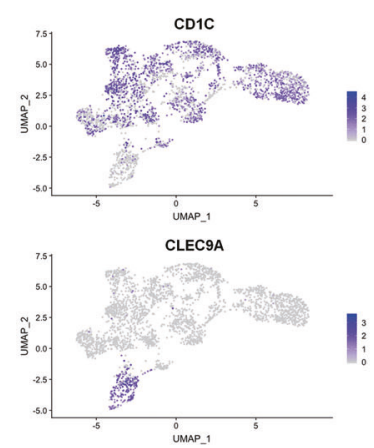

Fig. 3 The TME of LUAD is enriched with regulatory and exhausted $\mathrm{T}$ cells accompanied by increased $\mathrm{CD}^{+} \mathrm{C}^{+}$DCs. a UMAP plots of $\mathrm{T}$ cells derived from tumors and tumor-adjacent normal tissues with color-coded by different clusters (left) and different tissue origins (right). b UMAP plot of $\mathrm{T}$ cells derived from tumors and tumoradjacent normal tissues with color-coded by different T-cell subtypes.

d
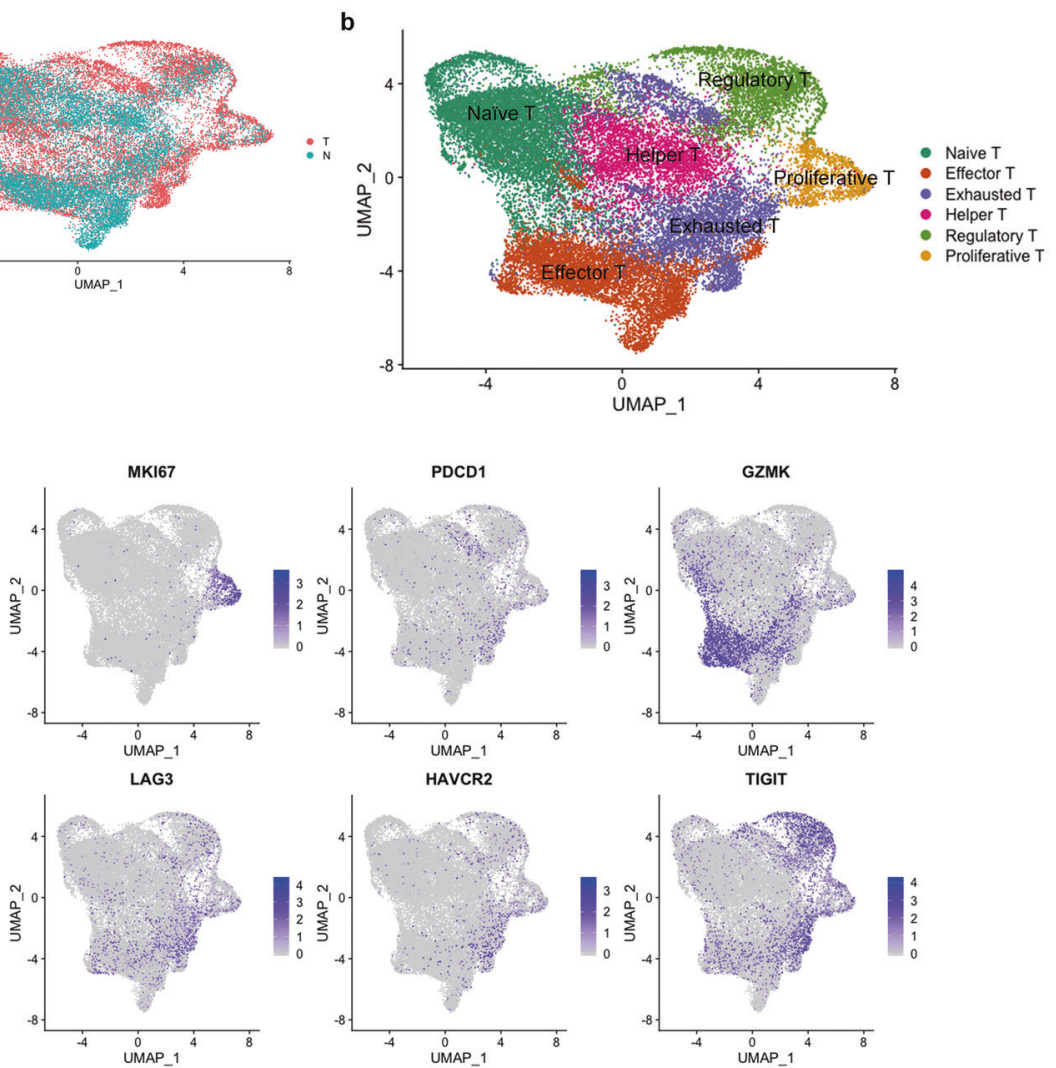

c The proportions of T-cell subtypes in each LUAD sample and tumoradjacent normal sample. d UMAP plots showing the expression signature of proliferating $\mathrm{T}$ cells in the TME, which express both effective and dysfunctional T-cell markers. e UMAP plots showing the expression of DC subtype markers, i.e., $C D 1 C\left(\mathrm{CD} 1 \mathrm{C}^{+} \mathrm{DC}\right)$ and CLEC9A $\left(\mathrm{CD} 141^{+} \mathrm{DC}\right)$, in all the DCs.

We pooled all the malignant cells (Fig. 1b) and constructed a single-cell pseudotime trajectory using monocle $3 \mathrm{R}$ package. Monocle 3 did a dimension reduction on all the tumor cells and ordered the cells based on their progression states. We manually set the root-state of the pseudotime trajectory within the cells of cluster 3 because they still expressed normal lung epithelium markers such as alveolar type 2 (AT2) cell marker SFTPC and Clara cell marker SCGB1A1, suggesting that they still remain the characteristics of normal lung epithelial cells (Supplementary Fig. S8). Then the pseudotime trajectory was obtained after calculating the pseudotime value of each cell (Fig. 5a). Using graph-test function of monocle3, we identified the genes upregulated in more advanced cells within the pseudotime trajectory (Supplementary Table S6). Although they were on the top of the list, long non-coding RNAs (LncRNAs) MALAT1 [33] and NEAT1 [34] have already been characterized as the hallmarks of metastasis in lung cancer [35] and other malignant tumors [36, 37]. We noted that additional altered genes encode structural proteins such as membrane channels (e.g., APQ3) and transporters (e.g.,
SLC34A2 and NPC2) that may be coupled to key regulators in tumor cells. We were particularly interested in studying those genes that are associated with cancer progression, TME signals, and poor prognosis such as LY6E [38], NAPSA [39], MUC1 [40, 41], ELF3 [42, 43], and FOS [44] (Fig. 5b). Some of them have been well characterized in lung cancers. For example, NAPSA is a common prognostic marker for LUAD. LY6E [38], MUC1 [45], and FOS [46] play important roles in immune escape and suppressive immune microenvironment in various types of cancers. In this study, we decided to focus on the role of ELF3, a transcriptional factor regulating lung epithelial development [47], which is implicated in airway inflammation [48] and mediates inflammatory signal and tumor progression in prostate cancer [49] and colorectal cancer [50].

To address the role of ELF3 in LUAD, we used the TCGA LUAD data $(n=515)$ and found that ELF3 was upregulated in tumor samples compared to the normal lung tissues (Fig. 5c). In addition, the gene set enrichment analysis (GSEA) of the TCGA dataset showed that the CCND1 and AKT upregulated gene sets were overrepresented in the 

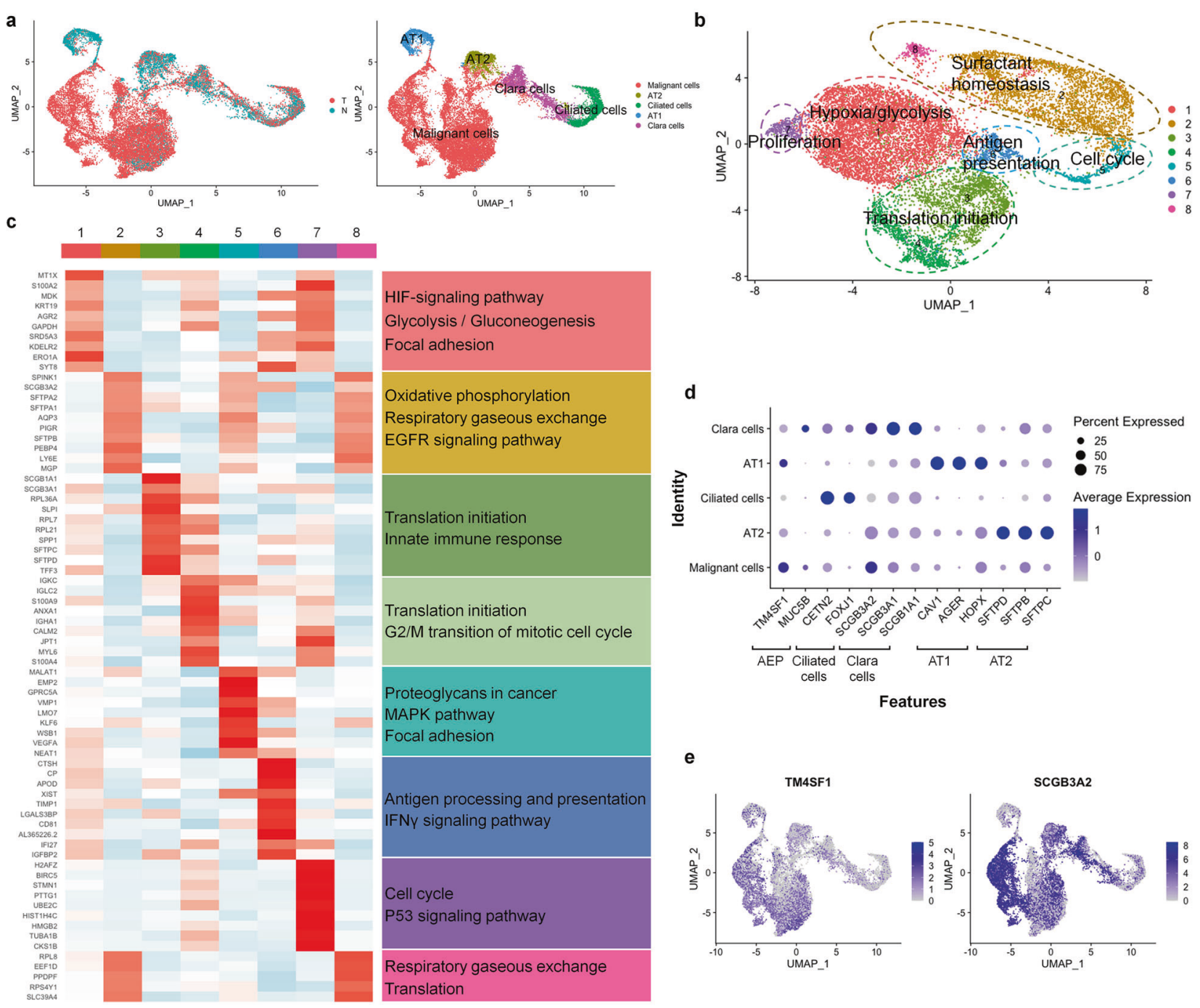

d

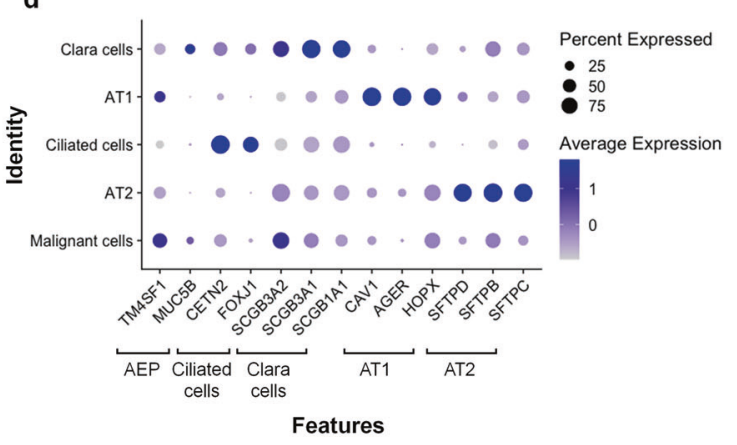

e

Fig. 4 Tumor cells within early-stage LUAD have heterogeneous gene expression signatures. a UMAP plots of 20,802 malignant cells and normal lung epithelial cells from all the tumor and normal samples with color labeled for different tissue origins (left) and cell types (right), respectively. b UMAP plots of 14,456 malignant cells showed 8 distinct tumor cell clusters. They can be grouped based on the functional enrichment analysis of their DEGs. The tumor cells displayed expression signatures of hypoxia/glycolysis, oxidative

tumors with higher ELF3 expression level (Fig. 5d). In conclusion, these results suggested that ELF3 was upregulated in more advanced LUAD cells and associated with upregulation of CCND1 and AKT in LUAD.

\section{ELF3 promotes tumor growth through PI3K/AKT/NF- KB signaling pathway in LUAD cells after IL1B induction}

ELF3 can regulate cell cycle and proliferation in NSCLC [42] and chemical-induced lung injury [51]. High expression of ELF3 also favors tumor growth through activation

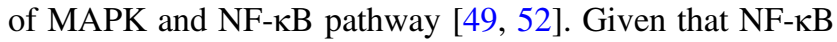

phosphorylation, translation, cell cycle, antigen presentation and proliferation. c Heatmap showing the expression of DEGs in each tumor cluster and their enriched KEGG pathways. d Expression of canonical lung epithelial markers in each cell type. In a given cell identify, the sizes of circles indicate percentage of the cells expressing each marker gene; The shades of blue indicate average expression of each gene. e UMAP plots showing elevated TM4SF1 and $S C G B 3 A 2$ expression in malignant cells than in normal lung epithelial cells.

pathway plays essential roles in activating survival genes for cancer cells as well as inflammatory response in immune cells [53, 54], we speculate that ELF3 may function as an important modulator of NF-kB pathway in LUAD. We first confirmed the upregulation of ELF3 at protein level in the tumor tissues compared to the normal lung tissues (Fig. 6a). We then examined the expression of ELF3 and NF- $\mathrm{KB}$ target genes in additional 12 primary LUAD tissues (T1T12, Supplementary Table S7) by quantitative real timePCR (qRT-PCR). We found that ELF3 was highly expressed in all the tumor tissues compared to the matched normal samples. Some NF-kB target genes such as CCND1, $B C L 2 L 1$ and $G A D D 45 B$ and ICAMI which are responsible 
a
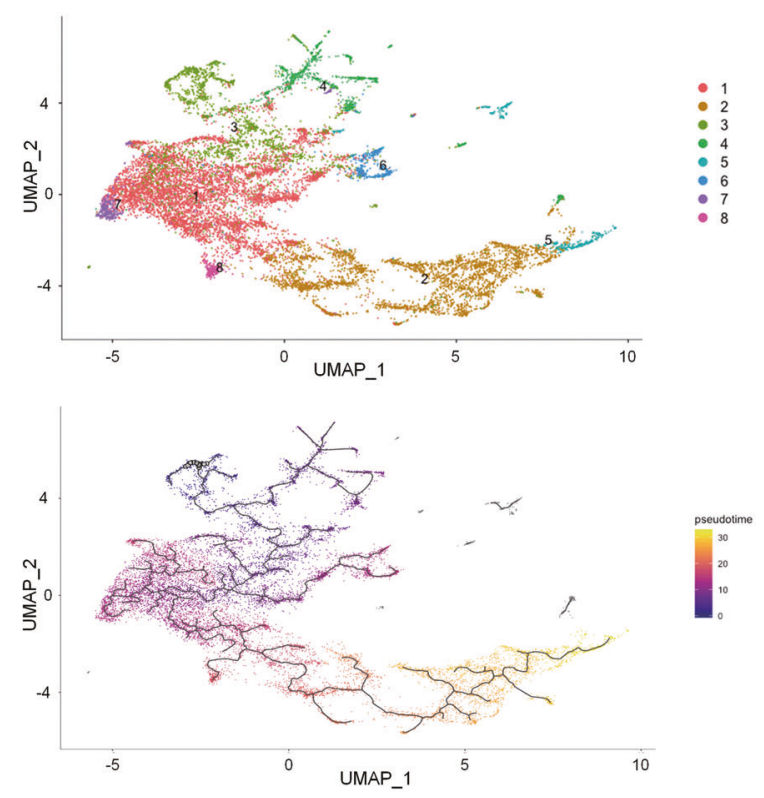

b

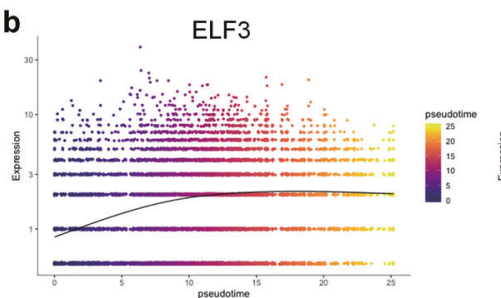

FOS

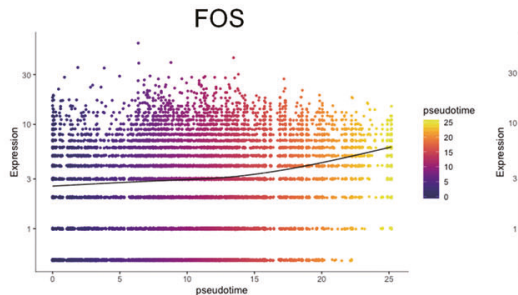

CCND1
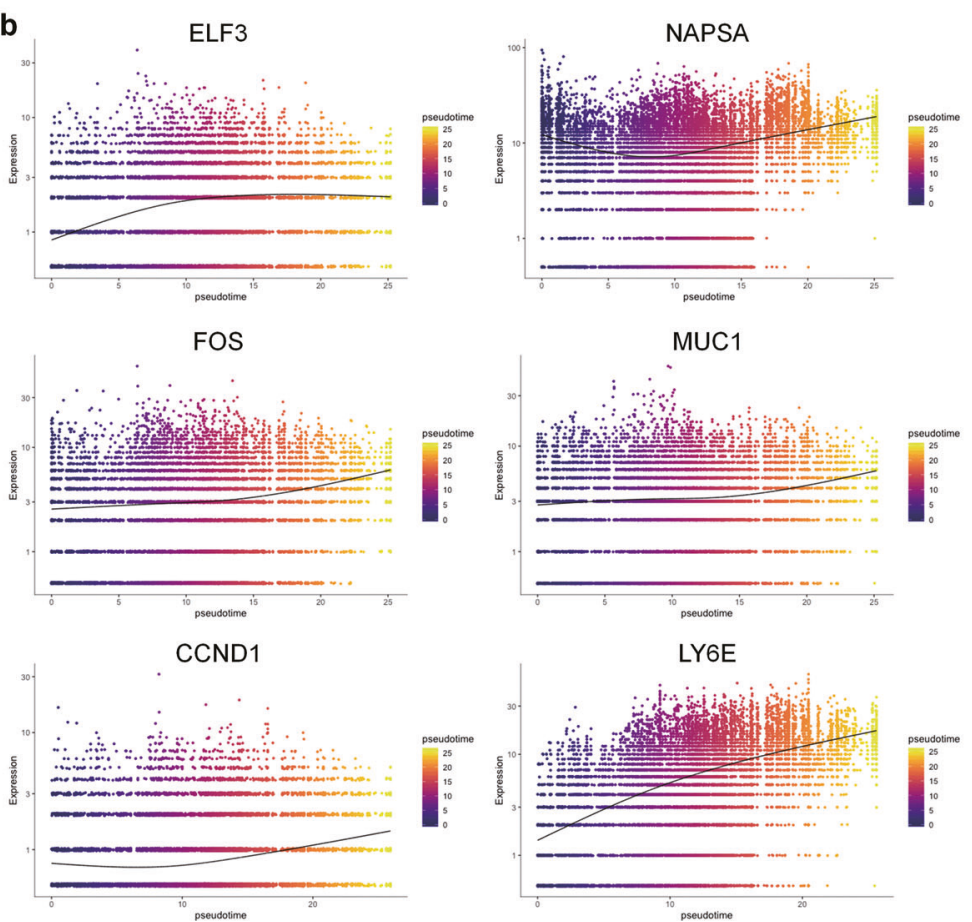

LY6E

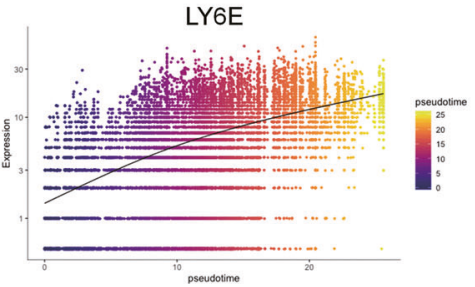

c

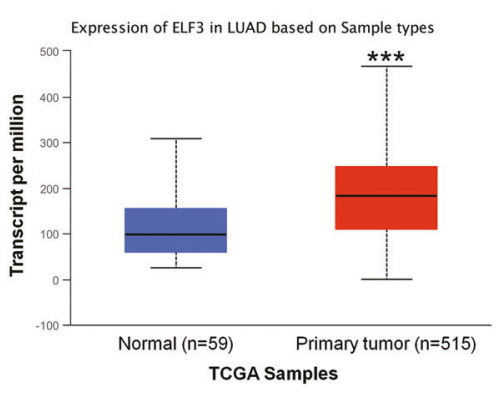

d
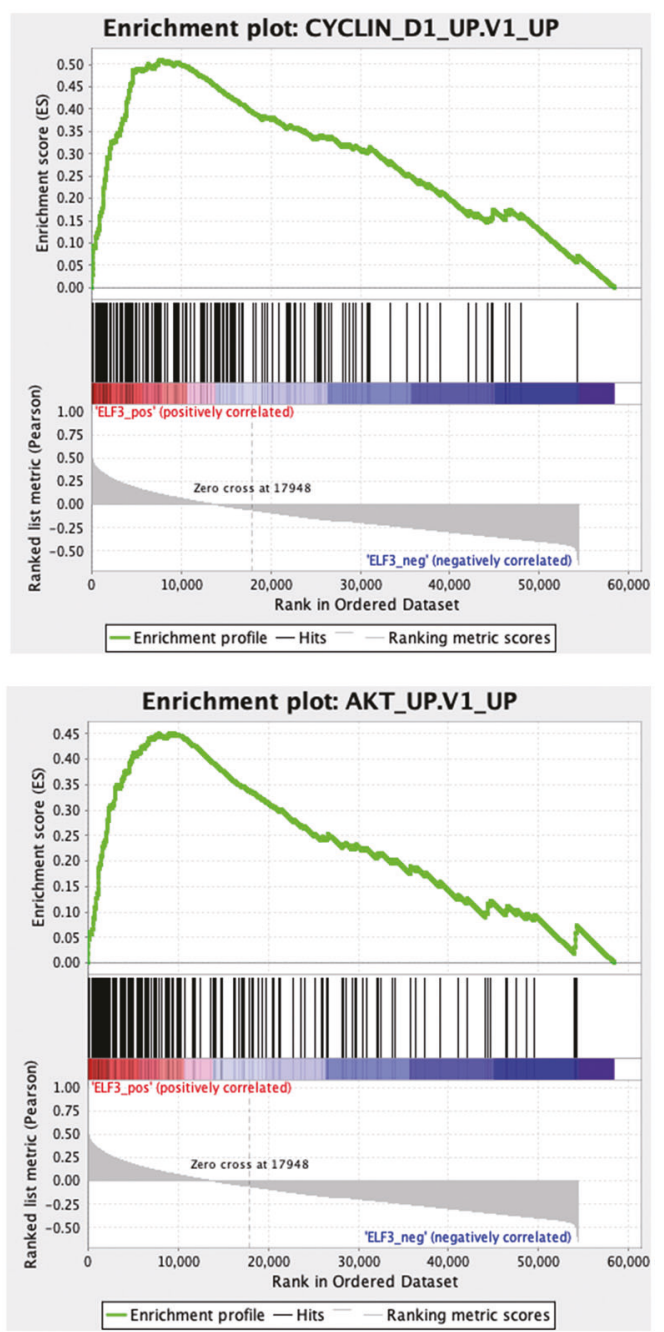

Fig. 5 Pseudotime trajectory analysis revealed the upregulated genes in more advanced tumor cells. a Pseudotime trajectory of all the malignant cells derived from 7 LUADs. Top: pseudotime trajectory tree of all the tumor cells with different colors indicating different clusters identified by Seurat. Bottom: all the tumor cells were colored by their assigned pseudotime values. b Jitter plots showing the expression level of the genes changing with pseudotime. ELF3, FOS, $M U C 1, C C N D 1$, and LY6E were among the most significantly

for proliferation, anti-apoptosis and adhesion were also upregulated in the tumor tissues (Fig. 6b).

Since other cell types in TME, especially immune cells, also highly express NF- $\mathrm{kB}$ target genes, we decided to use upregulated genes in cells with larger pseudotime. NAPSA, a LUAD prognostic marker, also have higher expression in more advanced tumor cells. c The TCGA LUAD $(n=515)$ dataset showing ELF3 was upregulated in LUAD samples compared to the normal lung tissues $(n=59)$. d Gene set enrichment analysis (GSEA) showing that the CCND1 and AKT upregulation gene sets were overrepresented in the tumors with high ELF3 level, indicating ELF3 level was associated with tumor cell proliferation and progression.

LUAD cell lines A549 and NCI-H1975 to further investigate the function of ELF3 in tumor cells. After knocking down ELF3 by siRNAs, the expression of NFKB1 remained unchanged (Fig. 6c). Some NF-kB target genes such as 
a

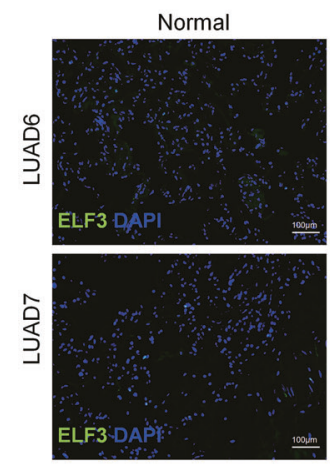

c

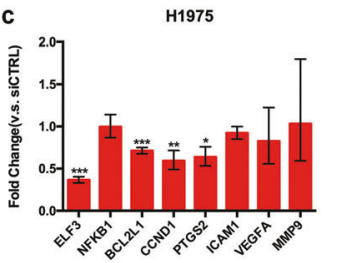

A549

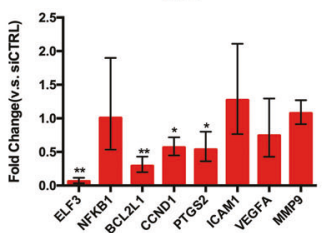

Fig. 6 ELF3 can be induced by IL1B and promotes tumor growth through PI3K/AKT/NF-кB pathway. a Representative immunostaining showed that ELF3 expression in LUAD6 and LUAD7 is higher than that in the matched normal lung tissues. b Expression of ELF3 and selected NF- $\mathrm{KB}$ target genes in additional 12 LUAD tumor samples by qRT-PCR. The plot showed that the median expression of ELF3 and $\mathrm{NF}-\kappa \mathrm{B}$ targeting genes related to proliferation and antiapoptosis are higher in the tumor tissues than their matched normal lung tissues. Colored dots refer to different individuals. c Expression of ELF3, NFKB1, and selected NF- $\mathrm{BB}$ target genes in NCI-H1975 (top) and A549 (bottom) cell lines by qRT-PCR after knockdown of ELF3 by siRNA (The controls were transfected with control siRNA (siCTRL)). In both cell lines, downregulation of ELF3 results in decreased expression of BCL2L1, CCND1, and PTGS2 which are responsible for anti-apoptosis, proliferation, and inflammation in tumor cells respectively. d Western blot showing the protein and

BCL2L1, CCND1, and PTGS2 were downregulated in both cell lines, while others responsible for angiogenesis (i.e., $V E G F A$ ) and metastasis (i.e., MMP9) remained unchanged (Fig. 6c). The reduced gene expression was resulted, at least in part, from the inactivation of PI3K/AKT/NF-KB pathway, as the phosphorylated proteins of PI3K, AKT, IKKa and P65 were all decreased after ELF3 knockdown (Fig. 6d).

It is known that pro-inflammatory cytokines secreted by immune cells such as IL1B and TNFa can activate NF- $\mathrm{KB}$ pathway in different cell types [55-57]. To determine whether ELF3 is involved the activation of NF- $\mathrm{KB}$ pathway in LUAD cells, we first treated A549 and NCI-H1975 cells with IL1B. We found elevated expression of ELF3 and the $\mathrm{NF}-\kappa \mathrm{B}$ target genes related to cell survival and
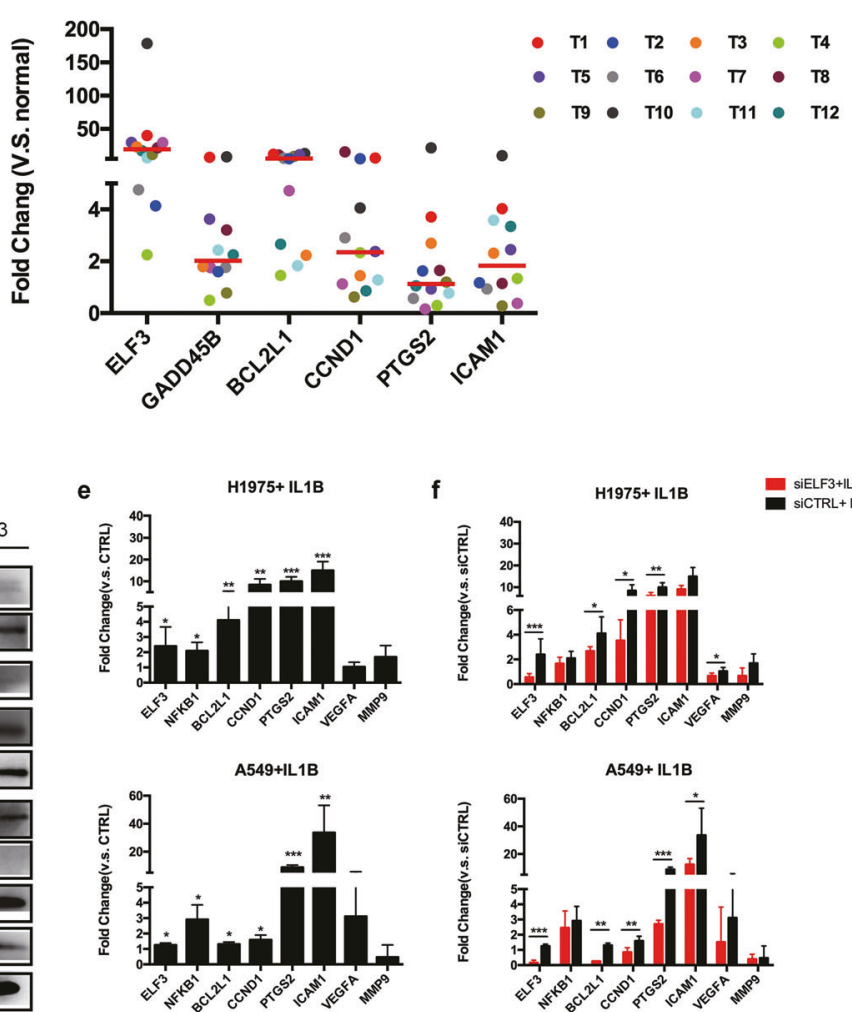

phosphorylation levels of the key components in PI3K/AKT/NF- $\kappa \mathrm{B}$ pathway in NCI-H1975 and A549 cells transfected with siELF3 or siCTRL. The phosphorylation levels of PI3K, AKT, IKKa, and P65 were decreased after ELF3 knockdown with unaltered total protein levels. e qRT-PCR showing the expression levels of ELF3, NFKB1,

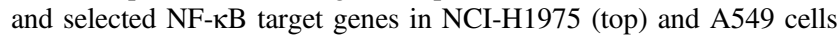
(bottom) treated with $10 \mathrm{ng} / \mathrm{ml}$ IL1B for $1 \mathrm{~h}$. The plots showed that the

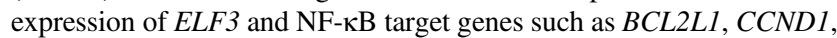
$P T G S 2$, and ICAM1 are increased in both cell lines after IL1B treatment. f qRT-PCR showing expression of ELF3, NFKB1, and the NF$\kappa \mathrm{B}$ target genes in NCI-H1975 (top) and A549 cells (bottom) which were transfected with siELF3 or siCTRL before being treated with $10 \mathrm{ng} / \mathrm{ml} \mathrm{IL1B}$ for $1 \mathrm{~h}$. The plots showed that IL1B induced upregulation of NF-KB target genes are compromised by ELF3 knockdown in both cell lines.

inflammation such as BCL2L1, CCND1, PTGS2, and ICAMI (Fig. 6e), which was compromised when ELF3 was knocked down in both cell lines (Fig. 6f). It should be noted that A549 and NCI-H1975 cells do not have CCND1 amplification according to the COSMIC cell line project database [58]. CCND1 amplification is seen in $~ 3.64 \%$ of the TCGA LUAD cohort. We inferred some copy number gains of CCND1 on chromosome 11 (Supplementary Fig. S6c), which may contribute to its overexpression in some tumor cells in addition to transcriptional regulation mediated by ELF3. Taken together, pro-inflammatory cytokine IL1B in the tumor microenvironment can upregulate ELF3 in LUAD tumor cells, which augments the activation of NF$\kappa \mathrm{B}$ pathway to favor tumor cell survival and growth. 
Our results implicated that ELF3 in LUAD tumor cells may serve as a novel therapeutic target to prevent tumor growth.

\section{Discussion}

The crosstalk with tumor cells and tumor microenvironment could promote tumor progression and metastases. However, few of the previous studies have investigated the transcriptome of lung tumor cells and their TME together, either in bulk or at single-cell level [18, 59-62]. Using dropletbased scRNA-seq technology (10X genomics), we analyzed different cell subpopulations in tumor samples simultaneously and comprehensively. We identified distinct subpopulations of tumor cells and their TME components, indicating that the heterogeneity in LUAD has already emerged at its early stage of tumorigenesis.

The immunal TME possesses either pro- or anti-tumor properties and has been widely studied for potential immunotherapy [63, 64]. We found that myeloid cells and $\mathrm{T}$ cells are the most abundant stromal cell types in the earlystage LUAD samples and their adjacent control tissues (Supplementary Fig. S2a). It is well known that alveolar macrophages, which do not express CD11b, are the most abundant cell population of resident immune cells [65]. Consistenly, at single-cell resolution, we show that lung resident macrophages are the most abundant stromal cell population, exhibiting high expression of $C D 68$ but not $C D 11 B$ (Fig. 2, Supplementary Figs. S2 and 3). Our results suggest that the overall immune TME is promoting tumor and immunosuppressive. Indeed, TAMs show high levels of gene expression enriched in the pathways which promote cell migration, angiogenesis, tumor progression, and inflammation (e.g., APOE and SPPl) (Fig. 2). Finally, the immunosuppressive state of TAMs in LUAD cannot be simply explained by M2 polarization, which is consistent with the previous reports that TAMs are not polarized to a distinct state of either M1 or M2 [16, 66].

Interestingly, we identified proliferating cell subtypes in both TAMs and $\mathrm{T}$ cells, which are characterized by high expression of proliferation marker MKI67. Although they have barely been reported in LUAD, proliferating TAMs and $\mathrm{T}$ cells were investigated in other tumor types. In breast cancer, proliferating TAMs were identified by proliferating cell nuclear antigen (PCNA) and found to be associated with tumor progression and poor prognosis $[67,68]$. These $\mathrm{PCNA}^{+}$proliferating TAMs in breast cancer also had high expression of MKI67 [69]. In the T cells, we identified a proliferating subtype that simultaneously express both effector T-cell and dysfunctional markers (Fig. 3c). A recent research reported a highly proliferating population of dysfunctional $\mathrm{T}$ cell in the TME of human melanoma [70]. We also found more regulatory and exhausted $\mathrm{T}$-cell clusters in the TME of LUAD, correlated with increased $\mathrm{CD}^{+} \mathrm{C}^{+} \mathrm{DC}$ population.

We investigated the role of an important modulator ELF3 in mediating interaction between TAMs and tumor cells in LUAD. ELF3 is an E26 transformation-specific (ETS) transcription factor highly expressed in epithelial-rich tissues. Its expression is relatively low in adult lung compared to fetal lung [71]. ELF3 was initially found to be overexpressed in HER2 positive breast cancer [72]. Soon after, its high expression was also reported in LUAD primary tumor and cell line A549 [71]. ELF3 is associated with poor prognosis in different tumor types including LUAD [42, 43], colorectal cancer [73], and hepatocellular carcinoma [74]. In prostate cancer, ELF3 regulates the NF- $\mathrm{KB}$ pathway after stimulated by inflammatory signals [49], suggesting that it may connect the TME signals and tumor progression. We demonstrate that some TAMs highly express inflammatory cytokine genes such as $I L 1 B$ (Fig. 2f) that activates NF- $\mathrm{KB}$ pathway. We hypothesize that IL1B released from TAMs triggers elevated expression of ELF3 in LUAD tumor cells. Knocking down ELF3 expression attenuates activation of NF- $\mathrm{KB}$ pathway genes in two LUAD cell lines A549 (KRAS mutation) and NCI-H1975 (EGFR mutation L858R), supporting the conclusion that inflammatory cytokines (e.g. IL1B) can regulate tumor cell proliferation and anti-apoptosis through ELF3 action.

Taken together, this work provides a valuable resource in understanding the heterogeneity and immunal cell profile in the early-stage LUAD harboring EGFR mutations in Asian patients. We identify crucial genes including ELF3 in mediating the interactions among tumor cells and their TME components, suggesting that ELF3 could be a therapeutic target in LUAD for future drug discovery.

\section{Material and methods}

\section{Preparation of single-cell suspensions from biopsies}

The tumor tissues and tumor-adjacent non-malignant lung tissues $(\sim 1.5 \mathrm{~cm} \times 1 \mathrm{~cm} \times 0.5 \mathrm{~cm})$ were resected after the surgeries and transported in DMEM/F12 medium (Gibco 11320082 ) on ice to the research facility within an hour. The details of tissue collection and dissociation protocol are described in Supplementary Materials and Methods.

\section{Single-cell RNA library construction, sequencing, and data analysis}

Single-cell suspensions $\left(\sim 1 \times 10^{6} / \mathrm{ml}\right)$ were submitted to 10X genomics Chromium Controller to generate single-cell GEMs (gel beads in emulsion). The constructed Chromium Single Cell 3' Library aiming for estimated 6000 cells for 
one sample following the manufacture's instruction $(10 \mathrm{x}$ genomics Chromium Single Cell 3' Library \& Gel Bead Kit v2). Then libraries were sequenced by Illumina Hiseq $X$ Ten platform. The raw sequence data reported in this paper have been deposited in the Genome Sequence Archive [75] in BIG Data Center [76], Beijing Institute of Genomics (BIG), Chinese Academy of Sciences. The access numbers are CRA001477 and CRA001963. Details of bioinformatics analysis of RNAseq data are provided in Supplemental Materials and Methods.

\section{Expression of $\mathrm{M} 1$ and $\mathrm{M} 2$ signature genes}

M1 and M2 signature genes (Supplementary Table S1) were curated form literature. The normalized weighted mean expression of those genes was calculated by Seurat's AddModuleScore and their correlation was suggested by Pearson correlation coefficient.

\section{GSEA analysis}

LUAD fpkm expression dataset $(n=585)$ and its phenotype labels were downloaded from UCSC Xena (https://xena browser.net). GSEA analysis was done by GSEA software [77] and gene set used was c2 gene set from Molecular Signatures Database (MSigDB).

\section{Immunostaining}

All the dissected tissues were fixed with 4\% PFA and embedded with paraffin. The $4 \mu \mathrm{m}$ tissue sections were cut and stained with hematoxylin and eosin for histology inspection. For immunohistolochemistry, anti-human CD3 (Servicebio GB11014), anti-human CD68 (Zsbio zm0060) were used. The immunofluorescence staining was performed as routine. The antibodies used were anti- human ELF3 (ABclonal A6371), anti-human CD68 (ZSGB ZM0060), anti-human KI67 (Servicebio GB14102), anti-human SFTPC (ABclonal A1835), anti-human SCGB3A1 (R\&D Systems MAB27901-SP) and anti-human SCGB3A2 (Abcam ab181853). The images were captured by Nikon ECLIPSE C1.

\section{Cell culture, shRNA interference, and IL1B treatment}

A549 and NCI-H1975 cells were cultured in RPMI-1640 medium (ThermoFisher Scientific 11875119) supplied with 10\% FBS (ThermoFisher Scientific 10099141) and 1\% penicillin and strepmycin (ThermoFisher Scientific 15140163 ) in $5 \% \mathrm{CO}_{2}$ at $37^{\circ} \mathrm{C}$. The siELF3 and siControl RNAs were pre-designed purchased from Thermofisher Technologies (4392420) and transfected using RNAiMAX transfection reagent (ThermoFisher Scientific 13778030) following the manufacturer's instruction. For IL1B treatment of both cell lines, recombinant human IL1B powder (R\&D systems 201-LB-010) was diluted with PBS $+1 \%$ FBS to $10 \mathrm{mg} / \mu \mathrm{l}$. $20 \mu \mathrm{g}$ of $(20 \mu \mathrm{l})$ IL1B were added to $2 \mathrm{ml}$ cultured medium of each well of six-well plate. After IL1B treatment for $1 \mathrm{~h}$, total RNA and protein of the cells were isolated for subsequent analysis.

\section{qRT-PCR}

The total RNAs were extracted by TRIzol reagent (Invitrogen 15596026). The reverse transcription was done using RevertAid RT Reverse Transcription Kit (ThermoFisher Scientific K1691) following the manufacture's protocol. The qPCR primers (Supplementary Table S2) were designed on https://sg.idtdna.com. The qPCR amplification was done using Taq polymerase (Takara RR820A) on Roche LightCycler 480 instrument. Fold change was calculated as $2^{(-\triangle \triangle \mathrm{CT})} ; \triangle \triangle \mathrm{CT}=\triangle \mathrm{CT}($ sample)- $\triangle \mathrm{CT}$ (control); $\triangle \mathrm{CT}=\mathrm{CT} \quad$ (gene)-CT (internal reference (B2M)).

\section{Western blotting}

The cells were lysed by RIPA lysis buffer (Epizyme PC101) followed by centrifuging at $12,000 \times g$ for $5 \mathrm{~min}$ at $4{ }^{\circ} \mathrm{C}$. Total protein was collected and stored in $-80^{\circ} \mathrm{C}$ until use. Western blotting was performed as routine. The primary antibodies used were anti-human ELF3 (ABclonal A6371), anti-human PI3K (Cell Signalling Technology 4249), antihuman P-PI3K (Cell Signalling Technology 4228), antihuman AKT (ABclonal A11030), anti-human P-AKT (Cell Signalling Technology 4060), anti-human P65 (Abcam ab16502), anti-human P-P65 (Abcam ab86299), anti-human IKKA (ABclonal A0422) and anti-human P-IKKA (ABclonal AP0546). Secondary antibody was goat antirabbit IgG $(\mathrm{H}+\mathrm{L})$ HRP (ABways AB0101).

\section{Web resources}

The raw sequence data reported in this paper have been deposited in the Genome Sequence Archive in BIG Data Center, Beijing Institute of Genomics (BIG), Chinese Academy of Sciences. They are publicly accessible at https://bigd.big.ac.cn/gsa.

Acknowledgements We thank Wei Jiang at Genergy Bio-technology (Shanghai) Co., Ltd and Minghui Li at Sinotech Genomics (Shenzhen) Co., Ltd for their contribution in library construction, sequencing and data analysis. The work was supported by National Program on Key Basic Research Project (973 Program 2015CB964702, 2015CB964601); National Natural Science Foundation of China (81570521, 81470244, 81622001, 81430026, 91642108, 31871288); 
Experimental Animal Research Fund, Science and Technology Commission of Shanghai Municipality (18140903900, 15140903900); Fundamental Research Funds for the Central Universities (Xianmin Zhu and Peng Zhang).

\section{Compliance with ethical standards}

Conflict of interest The authors declare that they have no conflict of interest.

Publisher's note Springer Nature remains neutral with regard to jurisdictional claims in published maps and institutional affiliations.

Open Access This article is licensed under a Creative Commons Attribution 4.0 International License, which permits use, sharing, adaptation, distribution and reproduction in any medium or format, as long as you give appropriate credit to the original author(s) and the source, provide a link to the Creative Commons license, and indicate if changes were made. The images or other third party material in this article are included in the article's Creative Commons license, unless indicated otherwise in a credit line to the material. If material is not included in the article's Creative Commons license and your intended use is not permitted by statutory regulation or exceeds the permitted use, you will need to obtain permission directly from the copyright holder. To view a copy of this license, visit http://creativecommons. org/licenses/by/4.0/.

\section{References}

1. Torre LA, Siegel RL, Jemal A. Lung cancer statistics. Adv Exp Med Biol. 2016;893:1-19.

2. Seow WJ, Matsuo K, Hsiung CA, Shiraishi K, Song M, Kim HN, et al. Association between GWAS-identified lung adenocarcinoma susceptibility loci and EGFR mutations in never-smoking Asian women, and comparison with findings from Western populations. Hum Mol Genet. 2017;26:454-65.

3. Hirsch FR, Scagliotti GV, Mulshine JL, Kwon R, Curran WJ Jr, $\mathrm{Wu} \mathrm{YL}$, et al. Lung cancer: current therapies and new targeted treatments. Lancet. 2017;389:299-311.

4. Cortot AB, Janne PA. Molecular mechanisms of resistance in epidermal growth factor receptor-mutant lung adenocarcinomas. Eur Respiratory Rev: Off J Eur Respiratory Soc. 2014;23:356-66.

5. Chittezhath M, Dhillon MK, Lim JY, Laoui D, Shalova IN, Teo YL, et al. Molecular profiling reveals a tumor-promoting phenotype of monocytes and macrophages in human cancer progression. Immunity. 2014;41:815-29.

6. Quail DF, Joyce JA. Microenvironmental regulation of tumor progression and metastasis. Nat Med. 2013;19:1423-37.

7. Hanahan D, Coussens LM. Accessories to the crime: functions of cells recruited to the tumor microenvironment. Cancer cell. 2012;21:309-22.

8. Wei SC, Duffy CR, Allison JP. Fundamental mechanisms of immune checkpoint blockade therapy. Cancer Disco. 2018;8:1069-86.

9. Okazaki T, Chikuma S, Iwai Y, Fagarasan S, Honjo T. A rheostat for immune responses: the unique properties of PD-1 and their advantages for clinical application. Nat Immunol. 2013;14: 1212-8.

10. June $\mathrm{CH}$, Sadelain M. Chimeric antigen receptor therapy. N Engl J Med. 2018;379:64-73.

11. Klein AM, Mazutis L, Akartuna I, Tallapragada N, Veres A, Li V, et al. Droplet barcoding for single-cell transcriptomics applied to embryonic stem cells. Cell. 2015;161:1187-201.
12. Zheng GX, Terry JM, Belgrader P, Ryvkin P, Bent ZW, Wilson $\mathrm{R}$, et al. Massively parallel digital transcriptional profiling of single cells. Nat Commun. 2017;8:14049.

13. Peterson VM, Zhang KX, Kumar N, Wong J, Li L, Wilson DC, et al. Multiplexed quantification of proteins and transcripts in single cells. Nat Biotechnol. 2017;35:936-9.

14. Hu P, Fabyanic E, Kwon DY, Tang S, Zhou Z, Wu H. Dissecting cell-type composition and activity-dependent transcriptional state in mammalian brains by massively parallel single-nucleus RNASeq. Mol Cell. 2017;68:1006-15.

15. Macosko EZ, Basu A, Satija R, Nemesh J, Shekhar K, Goldman $\mathrm{M}$, et al. Highly parallel genome-wide expression profiling of individual. Cells Using Nanoliter Droplets Cell. 2015;161: 1202-14.

16. Azizi E, Carr AJ, Plitas G, Cornish AE, Konopacki C, Prabhakaran S, et al. Single-cell map of diverse immune phenotypes in the breast tumor microenvironment. Cell. 2018;174:1293-308.

17. Peng J, Sun BF, Chen CY, Zhou JY, Chen YS, Chen H, et al. Single-cell RNA-seq highlights intra-tumoral heterogeneity and malignant progression in pancreatic ductal adenocarcinoma. Cell Res. 2019;29:725-38.

18. Lambrechts D, Wauters E, Boeckx B, Aibar S, Nittner D, Burton $\mathrm{O}$, et al. Phenotype molding of stromal cells in the lung tumor microenvironment. Nat Med. 2018;24:1277-89.

19. Chiossone L, Dumas PY, Vienne M, Vivier E. Natural killer cells and other innate lymphoid cells in cancer. Nat Rev Immunol. 2018;18:671-88.

20. Engblom C, Pfirschke C, Pittet MJ. The role of myeloid cells in cancer therapies. Nat Rev Cancer. 2016;16:447-62.

21. Psallidas I, Stathopoulos GT, Maniatis NA, Magkouta S, Moschos C, Karabela SP, et al. Secreted phosphoprotein-1 directly provokes vascular leakage to foster malignant pleural effusion. Oncogene. 2013;32:528-35.

22. Zheng P, Luo Q, Wang W, Li J, Wang T, Wang P, et al. Tumorassociated macrophages-derived exosomes promote the migration of gastric cancer cells by transfer of functional Apolipoprotein E. Cell Death Dis. 2018;9:434.

23. Murdoch $\mathrm{C}$, Lewis $\mathrm{CE}$. Macrophage migration and gene expression in response to tumor hypoxia. Int J cancer. 2005;117:701-8.

24. Goossens P, Rodriguez-Vita J, Etzerodt A, Masse M, Rastoin O, Gouirand V, et al. Membrane cholesterol efflux drives tumorassociated macrophage reprogramming and tumor progression. Cell Metab. 2019;29:1376-89.

25. Laoui D, Van Overmeire E, Di Conza G, Aldeni C, Keirsse J, Morias Y, et al. Tumor hypoxia does not drive differentiation of tumor-associated macrophages but rather fine-tunes the M2-like macrophage population. Cancer Res. 2014;74:24-30.

26. Huang AC, Postow MA, Orlowski RJ, Mick R, Bengsch B, Manne S, et al. T-cell invigoration to tumour burden ratio associated with anti-PD-1 response. Nature. 2017;545:60-65.

27. Lanitis E, Dangaj D, Irving M, Coukos G. Mechanisms regulating T-cell infiltration and activity in solid tumors. Ann Oncol. 2017;28:xii18-xii32.

28. Xu X, Rock JR, Lu Y, Futtner C, Schwab B, Guinney J, et al. Evidence for type II cells as cells of origin of K-Ras-induced distal lung adenocarcinoma. Proc Natl Acad Sci USA. 2012;109: 4910-5.

29. Sutherland KD, Song JY, Kwon MC, Proost N, Zevenhoven J, Berns A. Multiple cells-of-origin of mutant K-Ras-induced mouse lung adenocarcinoma. Proc Natl Acad Sci USA. 2014;111: 4952-7.

30. Guha A, Vasconcelos M, Cai Y, Yoneda M, Hinds A, Qian J, et al. Neuroepithelial body microenvironment is a niche for a distinct subset of Clara-like precursors in the developing airways. Proc Natl Acad Sci USA. 2012;109:12592-7. 
31. Reynolds SD, Reynolds PR, Pryhuber GS, Finder JD, Stripp BR. Secretoglobins SCGB3A1 and SCGB3A2 define secretory cell subsets in mouse and human airways. Am J Respiratory Crit Care Med. 2002;166:1498-509.

32. Zacharias WJ, Frank DB, Zepp JA, Morley MP, Alkhaleel FA, Kong J, et al. Regeneration of the lung alveolus by an evolutionarily conserved epithelial progenitor. Nature. 2018;555:251-5.

33. Ji P, Diederichs S, Wang W, Böing S, Metzger R, Schneider PM, et al. MALAT-1, a novel noncoding RNA, and thymosin beta4 predict metastasis and survival in early-stage non-small cell lung cancer. Oncogene. 2003;22:8031-41.

34. Zhang L, Zhang Z, Yu Z. Long non-coding RNA NEAT1 can predict various malignant tumour lympha node metastasis: a metaanalysis. Artif Cells Nanomed Biotechnol. 2019;47:2516-20.

35. Gutschner T, Hämmerle M, Eissmann M, Hsu J, Kim Y, Hung G, et al. The noncoding RNA MALAT1 is a critical regulator of the metastasis phenotype of lung cancer cells. Cancer Res. 2013;73:1180-9.

36. Sun Y, Ma L. New Insights into Long Non-Coding RNA MALAT1 in cancer and metastasis. Cancers. 2019;216:1-12.

37. Dong P, Xiong Y, Yue J, Hanley SJB, Kobayashi N, Todo Y, et al. Long non-coding RNA NEAT1: a novel target for diagnosis and therapy in human tumors. Front Genet. 2018;9:471.

38. AlHossiny M, Luo L, Frazier WR, Steiner N, Gusev Y, Kallakury $\mathrm{B}$, et al. Ly6E/K signaling to TGF $\beta$ promotes breast cancer progression, immune escape, and drug resistance. Cancer Res. 2016;76:3376-86.

39. Bishop JA, Sharma R, Illei PB. Napsin A and thyroid transcription factor-1 expression in carcinomas of the lung, breast, pancreas, colon, kidney, thyroid, and malignant mesothelioma. Hum Pathol. 2010;41:20-5.

40. Apostolopoulos V, Stojanovska L, Gargosky SE. MUC1 (CD227): a multi-tasked molecule. Cell Mol life Sci: CMLS. 2015;72:4475-500.

41. Ahmad R, Raina D, Joshi MD, Kawano T, Ren J, Kharbanda S, et al. MUC1-C oncoprotein functions as a direct activator of the nuclear factor-kappaB p65 transcription factor. Cancer Res. 2009;69:7013-21.

42. Wang H, Yu Z, Huo S, Chen Z, Ou Z, Mai J, et al. Overexpression of ELF3 facilitates cell growth and metastasis through PI3K/Akt and ERK signaling pathways in non-small cell lung cancer. Int $\mathbf{J}$ Biochem Cell Biol. 2018;94:98-106.

43. Enfield KSS, Marshall EA, Anderson C, $\mathrm{Ng} \mathrm{KW}$, Rahmati S, Xu $\mathrm{Z}$, et al. Epithelial tumor suppressor ELF3 is a lineage-specific amplified oncogene in lung adenocarcinoma. Nat Commun. 2019;10:5438.

44. Ouyang X, Jessen WJ, Al-Ahmadie H, Serio AM, Lin Y, Shih WJ, et al. Activator protein-1 transcription factors are associated with progression and recurrence of prostate cancer. Cancer Res. 2008;68:2132-44.

45. Bouillez A, Rajabi H, Jin C, Samur M, Tagde A, Alam M, et al. MUC1-C integrates PD-L1 induction with repression of immune effectors in non-small-cell lung cancer. Oncogene. 2017;36:4037-46.

46. Xiao G, Deng A, Liu H, Ge G, Liu X. Activator protein 1 suppresses antitumor T-cell function via the induction of programmed death 1. Proc Natl Acad Sci USA. 2012;109:15419-24.

47. Oliver JR, Kushwah R, Hu J. Multiple roles of the epitheliumspecific ETS transcription factor, ESE-1, in development and disease. Lab Investig; a J Tech methods Pathol. 2012;92:320-30.

48. Wu J, Duan R, Cao H, Field D, Newnham CM, Koehler DR, et al. Regulation of epithelium-specific Ets-like factors ESE-1 and ESE3 in airway epithelial cells: potential roles in airway inflammation. Cell Res. 2008;18:649-63.

49. Longoni N, Sarti M, Albino D, Civenni G, Malek A, Ortelli E, et al. ETS transcription factor ESE1/ELF3 orchestrates a positive feedback loop that constitutively activates NF-kappaB and drives prostate cancer progression. Cancer Res. 2013;73:4533-47.

50. Pocha K, Mock A, Rapp C, Dettling S, Warta R, Geisenberger C, et al. Surfactant expression defines an inflamed subtype of lung adenocarcinoma brain metastases that correlates with prolonged survival. Clin Cancer Res. 2020;26:2231-43.

51. Oliver JR, Kushwah R, Wu J, Pan J, Cutz E, Yeger H, et al. Elf3 plays a role in regulating bronchiolar epithelial repair kinetics following Clara cell-specific injury. Lab Investig. 2011;91:1514-29.

52. Chen H, Chen W, Zhang X, Hu L, Tang G, Kong J, et al. E26 transformation (ETS)specific related transcription factor3 (ELF3) orchestrates a positive feedback loop that constitutively activates the MAPK/Erk pathway to drive thyroid cancer. Oncol Rep. 2019;41:570-8.

53. Karin M, Cao Y, Greten FR, Li ZW. NF-kappaB in cancer: from innocent bystander to major culprit. Nat Rev Cancer. 2002;2:301-10.

54. Karin M. Nuclear factor-kappaB in cancer development and progression. Nature. 2006;441:431-6.

55. Grall F, Gu X, Tan L, Cho JY, Inan MS, Pettit AR, et al. Responses to the proinflammatory cytokines interleukin-1 and tumor necrosis factor alpha in cells derived from rheumatoid synovium and other joint tissues involve nuclear factor kappaBmediated induction of the Ets transcription factor ESE-1. Arthritis Rheumatism. 2003;48:1249-60.

56. Ozes ON, Mayo LD, Gustin JA, Pfeffer SR, Pfeffer LM, Donner DB. NF-kappaB activation by tumour necrosis factor requires the Akt serine-threonine kinase. Nature. 1999;401:82-5.

57. Apte RN, Krelin Y, Song X, Dotan S, Recih E, Elkabets M, et al. Effects of micro-environment- and malignant cell-derived interleukin-1 in carcinogenesis, tumour invasiveness and tumour-host interactions. Eur J Cancer (Oxf, Engl: 1990). 2006;42:751-9.

58. Tate JG, Bamford S, Jubb HC, Sondka Z, Beare DM, Bindal N, et al. COSMIC: the catalogue of somatic mutations in cancer. Nucleic Acids Res. 2018;47:D941-7.

59. Zhang Y, Wang Y, Lu M, Qiao X, Sun B, Zhang W, et al. Modular analysis of bioinformatics demonstrates a critical role for NF-kappaB in macrophage activation. Inflammation. 2014;37:1240-53.

60. Min JW, Kim WJ, Han JA, Jung YJ, Kim KT, Park WY, et al. Identification of Distinct Tumor Subpopulations in Lung Adenocarcinoma via Single-Cell RNA-seq. PloS ONE. 2015;10: e0135817.

61. Lavin Y, Kobayashi S, Leader A, Amir ED, Elefant N, Bigenwald $\mathrm{C}$, et al. Innate Immune Landscape in Early Lung Adenocarcinoma by Paired Single-cell analyses. Cell. 2017;169:750-65.

62. Guo X, Zhang Y, Zheng L, Zheng C, Song J, Zhang Q, et al. Global characterization of $\mathrm{T}$ cells in non-small-cell lung cancer by single-cell sequencing. Nat Med. 2018;24:978-85.

63. Altorki NK, Markowitz GJ, Gao D, Port JL, Saxena A, Stiles B, et al. The lung microenvironment: an important regulator of tumour growth and metastasis. Nat Rev Cancer. 2019;19:9-31.

64. Gajewski TF, Schreiber H, Fu YX. Innate and adaptive immune cells in the tumor microenvironment. Nat Immunol. 2013;14:1014-22.

65. Milette S, Fiset PO, Walsh LA, Spicer JD, Quail DF. The innate immune architecture of lung tumors and its implication in disease progression. J Pathol. 2019;247:589-605.

66. Mosser DM, Edwards JP. Exploring the full spectrum of macrophage activation. Nat Rev Immunol. 2008;8:958-69.

67. Mukhtar RA, Moore AP, Tandon VJ, Nseyo O, Twomey P, Adisa $\mathrm{CA}$, et al. Elevated levels of proliferating and recently migrated tumor-associated macrophages confer increased aggressiveness and worse outcomes in breast cancer. Ann surgical Oncol. 2012;19:3979-86.

68. Campbell MJ, Tonlaar NY, Garwood ER, Huo D, Moore DH, Khramtsov AI, et al. Proliferating macrophages associated with 
high grade, hormone receptor negative breast cancer and poor clinical outcome. Breast Cancer Res Treat. 2011;128:703-11.

69. Campbell MJ, Wolf D, Mukhtar RA, Tandon V, Yau C, Au A, et al. The prognostic implications of macrophages expressing proliferating cell nuclear antigen in breast cancer depend on immune context. PloS ONE. 2013;8:e79114.

70. Li H, van der Leun AM, Yofe I, Lubling Y, Gelbard-Solodkin D, van Akkooi ACJ, et al. Dysfunctional CD8 T cells form a proliferative, dynamically regulated compartment within human melanoma. Cell. 2019;176:775-89.

71. Tymms MJ, Ng AY, Thomas RS, Schutte BC, Zhou J, Eyre HJ, et al. A novel epithelial-expressed ETS gene, ELF3: human and murine cDNA sequences, murine genomic organization, human mapping to $1 \mathrm{q} 32.2$ and expression in tissues and cancer. Oncogene. 1997;15:2449-62.

72. Chang CH, Scott GK, Kuo WL, Xiong X, Suzdaltseva Y, Park JW, et al. ESX: a structurally unique Ets overexpressed early during human breast tumorigenesis. Oncogene. 1997;14:1617-22.
73. Wang JL, Chen ZF, Chen HM, Wang MY, Kong X, Wang YC, et al. Elf3 drives $\beta$-catenin transactivation and associates with poor prognosis in colorectal cancer. Cell Death Dis. 2014;5: e1263.

74. Zheng L, Xu M, Xu J, Wu K, Fang Q, Liang Y, et al. ELF3 promotes epithelial-mesenchymal transition by protecting ZEB1 from miR-141-3p-mediated silencing in hepatocellular carcinoma. Cell Death Dis. 2018;9:387.

75. Wang Y, Song F, Zhu J, Zhang S, Yang Y, Chen T, et al. GSA: genome sequence archive. Genomics, Proteom Bioinforma. 2017;15:14-8.

76. BIG Data Center Members. Database resources of the BIG data center in 2019. Nucleic Acids Res. 2019;47:D8-14.

77. Subramanian A, Tamayo P, Mootha VK, Mukherjee S, Ebert BL, Gillette MA, et al. Gene set enrichment analysis: a knowledge-based approach for interpreting genome-wide expression profiles. Proc Natl Acad Sci USA. 2005;102: $15545-50$. 